\title{
FLARE STARS DATABASE
}

\author{
M. TSVETKOV ${ }^{1 \dagger}$, M. CHUKOVA ${ }^{2}$ and K. TSVETKOVA ${ }^{1}$ \\ ${ }^{1}$ Department of Astronomy, Bulgarian Academy of Sciences, Tsarigradsko Shose 72, \\ BG-1784 Sofia, Bulgaria \\ ${ }^{2}$ Computer Centre of Physics, Bulgarian Academy of Sciences, Tsarigradsko Shose \\ 72, BG-1784 Sofia, Bulgaria
}

The important role of flare stars (UV Ceti type variables) in astrophysics is due to the fact that the flare activity is not only typical for red stars with small masses but is a necessary stage during their evolution. The flare star search in stellar aggregates has led to the accumulation of rich observational material allowing us to look for statistical regularities in star formation and evolution. At present, there are more than 1500 known flare stars in the Galaxy; these were discovered mainly during the last 30 years. Most results of the long term monitoring with widefield telescopes are listed in existing catalogues of flare stars in stellar aggregates and in the solar neighbourhood. These catalogues and their machine-readable versions were the basis for the present database of flare stars in the Galaxy.

The programme package FLAREBASE represents a flexible, user-friendly environment for easy access and work on data arrays with independent contents. The most basic functions for management of databases are implemented. The package is written in $\mathrm{C}++$, a language which has major advantages for the creation of effective object code. Thus, the programme is convenient for installation and use even on personal computers with limited memory and hard disk space. Its executable code of $80 \mathrm{kB}$ allows the use of most of the available memory for the data arrays that hold the characteristics of the stars or other objects. As a result the service, effectiveness, and flexibility are quite good. The programme response is fast and is relatively independent of the hardware environment because indexed files, containing the sorted data, are stored on the hard disk and replaced only in case of data change. Therefore, during use the rearranged data from the smallest to the biggest stellar catalogues can be displayed on the screen immediately, even in the case of slow computer systems. Thus, the effectiveness for extracting data with certain criteria increases.

When starting the programme four options can be chosen:

1) a: creation and insertion of information arrays;

2) d: deletion of information arrays from the archive of FLAREBASE;

3) m: modification of the table format of chosen information arrays;

4) w: work with the FLAREBASE catalogues.

\footnotetext{
${ }^{\dagger}$ Research Fellow of the Alexander von Humboldt Foundation at the Astronomical Institute of the Westfälishe Wilhelms Universität, Münster, Germany.
} 
The following functions for work with the data are provided:

EDIT editing of a chosen record from a given data array;

SORT record sorting in a chosen field;

SEARCH extraction of all records, fulfilling previously given search criteria;

ADD line addition;

DEL line deletion;

PRINT display by printing.

FLAREBASE now includes the data on UV Ceti stars from the following original catalogues or their existing machine-readable versions:

1) General Catalogue of Variable Stars (Kholopov P.N. [ed.] 1985, Nauka, Moscow);

2) New Catalogue of Suspected Variable Stars (Kholopov P.N. [ed.] 1982, Nauka, Moscow);

3) 67th, 68th, 69th, 70th and 71st Name-Lists of Variable Stars (Kholopov P.N., Samus, N.N., Kazarovets, E.V., Perova, N.B., 1985, IBVS No. 2681; Kholopov, P.N., Samus, N.N., Kazarovets, E.V., Kireeva, N.N., 1987, IBVS No. 3058; Kholopov, P.N., Samus, N.N., Kazarovets, E.V., Florov, M.S., Kireeva, N.N., 1989, IBVS No. 3323; Kazarovets, E.V., Samus, N.N., 1990, IBVS No. 3530; Kazarovets, E.V., Samus, N.N., Gorannskij 1993, IBVS No 3840);

4) A Catalogue of Flare Stars in the Solar Vicinity (Shahovskaya, N.I., 1978, in 'Flares in Red Dwarf Stars', ed. R. Gershberg, Nauka, Moscow; Pettersen, B.R. 1976, Catalogue of Flare Star Data, Institute of Theoretical Astrophysics, Blindern-Oslo, Report No. 46; Semkov, E.N., Tsvetkov, M.K., Stavrev, K.Y., 1988, Preprint of NAO Rozhen);

5) Tonantzintla Catalogue of Flare Stars in the Pleiades Region (Haro, G., Chavira, E., Gonzales, G., 1982, Bolletin Instituto Tonantzintla, 3, No. 1, 3; Tsvetkov, M.K., Stavrev, K.Y., Tsvetkova, K.P., 1993, New Machine-readable version of the Tonantzintla Catalogue of Flare Stars in the Pleiades Region, in preparation);

6) A Catalogue of Flare Stars in the Orion Nebula Region (Natsvlishvili, R.Sh., 1991, Astrofizika, 31, 107);

7) A Catalogue of Flare Stars in the Cygnus Region (Tsvetkov, M.K., Tsvetkova, K.P., 1990, Proceedings of the IAU Symposium 137, eds. L. Mirzoyan, B. Pettersen, M. Tsvetkov).

8) A Catalogue of Flare Stars in the Praesepe Region (Tsvetkova, K.P., Tsvektov, M.K., Jankovics, I., 1991, Preprint of the Astronomical Institute Münster);

9) A Catalogue of Flare Stars in the Coma Berenices Cluster (Erastova, L.K., 1981, Thesis, Yerevan);

10) A Catalogue of Flare Stars in the Cassiopeia Region (Poulakos C., 1977, Praktika, Academy of Athens, 52; Poulakos, C., 1977, Acta Astronomica 27, 87; Poulakos, C., 1977, Astron. Astrophys. Suppl. 27, 429);

11) A Catalogue of Flare Stars in the Camelopardalis Region (Poulakos, C., 1977, Praktika, Academy of Athens, 51);

12) Bibliographic Catalogue of Variable Stars (Wenzel, W., 1981, Bull. Inform. CDC, No. 20, p. 105);

13) References to the General Catalogue of Variable Stars;

14) References to the New Catalogue of Suspected Variable Stars;

15) References to the 67th - 71st Name-lists of Variable Stars. 
In process of preparation to be included are the Catalogues of Flare Stars in the Tauri Dark Clouds and NGC 2264. The programme was demonstrated during this meeting and is available on a 3.5" (1.44 Mb) diskette from the authors.

\section{Acknowledgements}

We would like to thank W. Seitter for ongoing attention to our work and for making it possible to work in the Münster Astronomical Institute, P. Ivanov for the support and R. Duemmler for helpful suggestions and comments. M.T. acknowledges support from the Alexander von Humboldt Foundation. 